\title{
Localización intraoperatoria de las recurrencias en cáncer de tiroides mediante la inyección de azul de metileno guiada por ecografía
}

\author{
Intraoperative localization of recurrences in thyroid cancer by \\ ultrasound-guided injection of methylene blue
}

\author{
Ximena Franco-Castañeda ${ }^{1} \mathbb{D}$, Andrés Ignacio Chala-Galindo ${ }^{2} \mathbb{D}$ \\ 1 Médica, residente de Cirugía General, Universidad de Caldas, Manizales, Colombia. \\ 2 Medico, especialista en Cirugía general y Cirugía de Cabeza y Cuello; jefe, Servicio de Cirugía de Cabeza y Cuello; profesor aso- \\ ciado de Cirugía, Facultad de Salud, Universidad de Caldas, Manizales, Colombia.
}

\section{Resumen}

Introducción. La cirugía para extirpación de metástasis en un cuello previamente intervenido afronta un reto para lograr una resección exitosa. El presente estudio pretende demostrar la utilidad de la técnica de inyección de azul de metileno, guiada por ecografía, para la localización intraoperatoria de lesiones recurrentes en cáncer de tiroides, para facilitar su resección.

Métodos. Se realizó un estudio observacional, descriptivo y retrospectivo, en pacientes reintervenidos por recurrencia de carcinoma diferenciado de tiroides, durante un periodo de dos años y medio. Se utilizó la inyección intratumoral de azul de metileno guiada por ecografía para su identificación intraoperatoria de recurrencia. Se hizo análisis de variables demográficas y clínicas.

Resultados. Este estudio incluyó 10 procedimientos en 9 pacientes, 77,8 \% mujeres, con una media de edad de 54 años. Todos tenían un nivel de tiroglobulina detectable y elevado antes de la intervención; posteriormente, el 89 $\%$ presentó un descenso y el $33 \%$ una adecuada respuesta bioquímica. La técnica agregó 10 minutos al tiempo quirúrgico. En el $100 \%$ se identificaron de manera intraoperatoria los ganglios marcados; el promedio de ganglios resecados fue de 12, de los cuales, 6 fueron positivos, todos con carcinoma papilar de tiroides. Esta técnica se consideró de gran utilidad y de bajo costo en todos los casos.

Discusión. Esta técnica se muestra como una estrategia efectiva para la identificación intraoperatoria de las recurrencias locorregionales en carcinoma de tiroides, permitiendo una disección ganglionar exitosa, disminuyendo complicaciones, tiempo quirúrgico y, especialmente, costos frente a otras intervenciones.

Palabras clave: cáncer papilar tiroideo; recurrencia local de neoplasia; escisión del ganglio linfático; reoperación; azul de metileno; ultrasonografía.

Fecha de recibido: 15/09/2020 - Fecha de aceptación: 15/03/2021 - Fecha de publicación en línea: 10/06/2021

Correspondencia: Ximena Franco-Castañeda, Carrera 29 \# 69-49, Manizales, Colombia. Teléfono: 3117352578.

Correo electrónico: xime9497@hotmail.com

Citar como: Franco-Castañeda X, Chala-Galindo Al. Localización intraoperatoria de las recurrencias en cáncer de tiroides mediante la inyección de azul de metileno guiada por ecografía. Rev Colomb Cir. 2021;36:611-19. https://doi.org/10.30944/20117582.856 Este es un artículo de acceso abierto bajo una Licencia Creative Commons - BY-NC-ND https://creativecommons.org/licenses/by-ncnd/4.0/deed.es 


\begin{abstract}
Introduction. The approach of a previously operated neck for metastasis resection faces a challenge to achieve a successful resection. The present study aims to demonstrate the usefulness of the ultrasound-guided injection of methylene blue technique for the intraoperative localization of recurrent lesions in thyroid cancer to facilitate their resection.
\end{abstract}

Methods. An observational, descriptive and retrospective study was conducted in patients reoperated for recurrences of differentiated thyroid carcinoma over a period of two and a half years, using ultrasound-guided intratumoral injection of methylene blue for its intraoperative identification. An analysis of demographic and clinical variables was carried out and its advantages over other methods were identified.

Results. This study included 10 procedures in nine patients, $77.8 \%$ women and $22.2 \%$ men, with a mean age of 54 years. All had a detectable and elevated thyroglobulin level before the intervention, $89 \%$ had a decrease in its level and 33\% had an adequate biochemical response. The technique added 10 minutes to the surgical time. All marked lymph nodes were identified intraoperatively. The average number of lymph nodes resected was 12 , of which six were positive, all with papillary thyroid carcinoma. It was considered of great utility and low cost in all cases.

Discussion. This technique shows to be an effective strategy for the intraoperative identification of locoregional recurrences in thyroid carcinoma, allowing a successful lymph node dissection, reducing complications, surgical time and especially costs compared to other interventions.

Keywords: papillary thyroid cancer; local recurrence of neoplasia; lymph node excision; reoperation; methylene blue; ultrasonography.

\section{Introducción}

El carcinoma de tiroides es considerado el tumor endocrino más frecuente, siendo más prevalente en el género femenino, con una relación de 4:1 comparado con el género masculino ${ }^{1}$. El carcinoma papilar corresponde al tipo histológico más frecuente, constituyendo alrededor del $85 \%$ de los $\operatorname{casos}^{2}$.

En general, el cáncer diferenciado de tiroides es de buen pronóstico, pero se han encontrado recidivas locorregionales hasta en un $30 \%$ de los casos, con compromiso del compartimento lateral del cuello en más del $50 \%$ de las veces, mientras que las recaídas en el compartimento central son inferiores al $30 \%{ }^{3,4}$.

Desde la introducción de la ecografía como método de rutina para el seguimiento de los pacientes, ha sido posible una mayor detección de recidivas cervicales ganglionares inferiores a 1 centímetro de diámetro, que generalmente no se detectan a la palpación, ya que la ultrasonografía de alta resolución puede identificar lesiones desde los 2 a 3 mm de diámetro ${ }^{5,6}$.
En el momento de operar un cuello previamente intervenido, es importante tener en cuenta que existirán algunas limitaciones en el acto quirúrgico, debido a la distorsión de las estructuras anatómicas generada por fibrosis, procesos inflamatorios locales, edema, friabilidad de los tejidos y obliteración de los puntos de referencia por tejido cicatricial y, por lo tanto, dificultan la identificación de la lesión objeto de la resección, disminuyendo la probabilidad de éxito del procedimiento ${ }^{7,8,9}$. Además, es necesario resaltar el incremento del riesgo de complicaciones intraoperatorias, destacándose la lesión del laríngeo recurrente y de las glándulas paratiroides ${ }^{10}$.

En los últimos años ha sido posible implementar una serie de medidas con el fin de disminuir estas dificultades. Dentro de estas se destaca el papel de la ecografía intraoperatoria para localizar las lesiones metastásicas; sin embargo, se ha visto que la tasa de resecciones fallidas es alta ${ }^{11,12}$.

Como estrategia para incrementar el éxito quirúrgico en la extirpación de las recaídas tumorales y para disminuir las complicaciones de la cirugía, 
se ha desarrollado la técnica ROLL (radioguided occult lesion localization) ${ }^{8}$, la cual consiste en la inyección intralesional preoperatoria, guiada por imágenes como el ultrasonido o la tomografía computarizada, de un radiotrazador, por lo general, albúmina macroagregada marcada con Tecnecio-99, con lo cual se obtiene la concentración del radiotrazador en el tejido diana y, de manera intraoperatoria mediante una gamma sonda portátil, se detecta el lugar que presente más radioactividad y se procede a su resección ${ }^{13}$.

Otra técnica utilizada es la inyección intralesional de colorantes azules, guiada por ultrasonido. En un estudio publicado por Sippel et al., en la Universidad de California en el año 2009, utilizaron tres tintes azules diferentes (linfazurin, índigo carmín y azul de metileno) para la ubicación de adenopatías sospechosas, que por su tamaño no se podían identificar al examen físico, en diez reoperaciones por recidiva de carcinoma papilar de tiroides, evidenciando la efectividad y el éxito en cuanto a la resección de las lesiones metastásicas en todos los pacientes ${ }^{14}$. Posteriormente, el mismo grupo publicó otro estudio en una cohorte más grande, incluyendo 53 cirugías, en el que se ratificó que se trataba de un método eficaz y seguro ${ }^{15}$.

El objetivo de este estudio fue demostrar la utilidad de la técnica de inyección intralesional de azul de metileno, guiada por ultrasonografía, para la marcación y resección de las recidivas de carcinoma papilar de tiroides. Con la implementación de esta técnica se buscó incrementar el éxito quirúrgico, así como disminuir el tiempo operatorio y los costos del procedimiento.

\section{Métodos}

Se realizó un estudio descriptivo y retrospectivo, en el que se incluyeron los individuos llevados a reintervención por carcinoma de tiroides, para la localización de las lesiones metastásicas en cuello con la técnica de inyección intralesional de azul de metileno bajo guía ecográfica, entre febrero de 2018 y agosto de 2020, por el servicio de Cirugía de Cabeza y Cuello de la Facultad de Salud de la Universidad de Caldas, en la ciudad de Manizales, Colombia.
Para la búsqueda de recidiva locorregional se utilizó ultrasonografía, tomografía computarizada, niveles de tiroglobulina en sangre y citología por aspiración con aguja fina (BACAF) de la lesión sospechosa. Se reoperaron los pacientes con recidivas comprobadas por diagnóstico histológico, en al menos un ganglio linfático positivo para metástasis.

En sala de cirugía, con el paciente previamente anestesiado, se practicó la ecografía por parte del cirujano de cabeza y cuello con suficiente entrenamiento y experiencia en ultrasonografía (Figura 1). Se localizaron los ganglios patológicos y se compararon con las imágenes diagnósticas previas que aportaba el paciente, verificando la identificación adecuada de las lesiones a intervenir.

En el nódulo patológico se realizó la inyección de 0,5 a $1 \mathrm{ml}$ de azul de metileno diluido en $5 \mathrm{a}$ $10 \mathrm{ml}$ de solución salina normal (SSN 0,9\%), bajo guía ecográfica en tiempo real (Figura 2). La co-

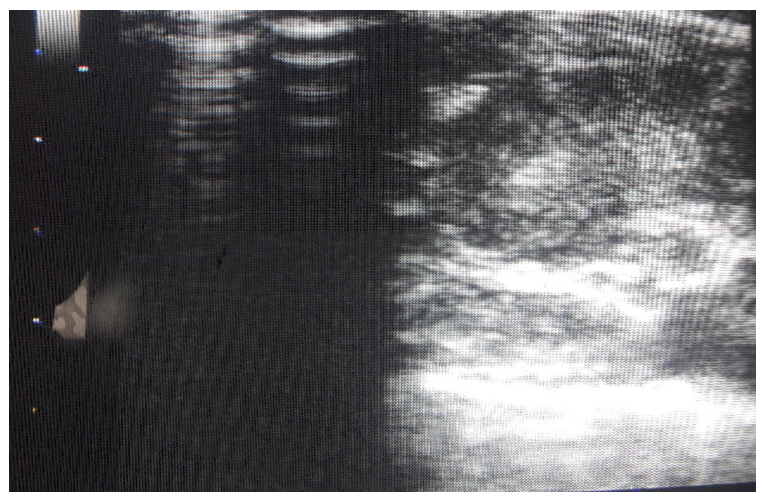

Figura 1. Localización ecográfica del ganglio. Fuente: Archivo doctor Andrés Ignacio Chala Galindo.

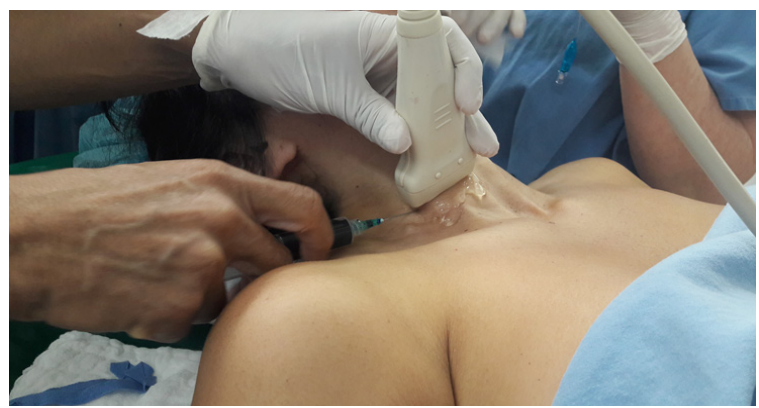

Figura 2. Inyección intratumoral de azul de metileno bajo guía ecográfica. Fuente: Archivo doctor Andrés Ignacio Chala Galindo 
loración funcionó de una manera eficaz para teñir el ganglio linfático objeto de resección (Figura 3). Después de la extracción de la pieza quirúrgica se envió al laboratorio de patología.

Se llevó a cabo una revisión de las historias clínicas de los pacientes identificados y se consignaron los datos en una base de datos en Microsoft $^{\circledR}$ Excel $^{\circledR}$. Se practicó un análisis descriptivo teniendo en cuenta variables demográficas, como edad y sexo; variables clínicas, como niveles de tiroglobulina; estudios imagenológicos, antes y después de la cirugía, así como citología de biopsia por aspiración con aguja fina (BACAF), identificación ecográfica e intraoperatoria del ganglio sospechoso teñido con el colorante, el reporte de la patología y las complicaciones del procedimiento.

\section{Resultados}

Entre el primero de febrero de 2018 y el 31 de agosto de 2020, se realizaron diez intervenciones quirúrgicas con esta técnica de inyección intratumoral de azul de metileno guiada por ecografía, en nueve pacientes con metástasis locorregional, a quienes previamente se les había realizado tiroidectomía total por carcinoma papilar de tiroides y vaciamiento ganglionar central o lateral del cuello, uno de los cuales fue operado en dos oportunidades en ese periodo.

Se encontró que todos los pacientes tenían carcinoma papilar de tiroides. Las edades de los pacientes oscilaron entre 42 y 72 años, con un promedio de 54,1 años. La distribución por sexo

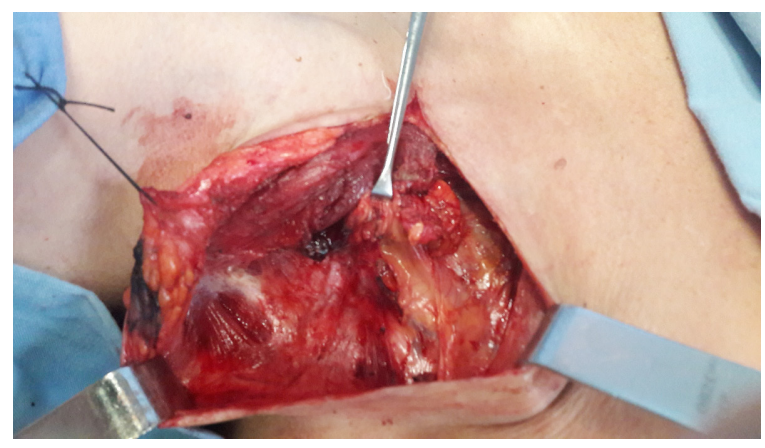

Figura 3. Identificación intraoperatoria del ganglio teñido con azul de metileno. Fuente: Archivo doctor Andrés Ignacio Chala Galindo. correspondió a 7 mujeres (77,8 \%) y 2 hombres $(22,2 \%)$. En cuanto al sitio de localización de la recaída, en dos de los casos se ubicó en el compartimento central cervical, siete en el lateral y uno tuvo recaída en ambos compartimentos.

En todos se identificó la recaída mediante el seguimiento de rutina, con niveles de tiroglobulina séricos, ecografía cervical y tomografía computarizada; dos de los casos tenían PET-SCAN preoperatoria. Todos los pacientes tenían un nivel de tiroglobulina detectable al inicio del estudio (promedio $115 \mathrm{ng} / \mathrm{ml}$ ). Cuando se detectó la presencia de adenopatías sospechosas, se confirmó mediante biopsia por aspiración con aguja fina (BACAF).

En todos los casos fue posible la identificación intraoperatoria y la resección de los ganglios teñidos con el colorante azul. La localización se consideró exitosa y de gran utilidad por parte del cirujano en todos los casos. El número promedio de ganglios resecados en cada reintervención fue de 12 (rango 1 a 47), de los cuales un promedio de 6 ganglios (rango 1 a 26) fueron positivos para metástasis de carcinoma papilar. En todos se comprobó mediante el reporte de la patología que el grupo ganglionar identificado en el acto quirúrgico fue positivo para malignidad.

La inyección del azul de metileno agregó aproximadamente 10 minutos al tiempo quirúrgico en la sala de cirugía previo a la incisión. No hubo complicaciones relacionadas con la inyección del azul de metileno. Las dificultades durante la cirugía fueron dadas por los hallazgos de fibrosis cicatricial en pacientes previamente intervenidos, lo cual se superó con la identificación de los ganglios teñidos con el azul de metileno, para orientar la resección de los mismos. En ningún caso se evidenciaron complicaciones en el posoperatorio, no hubo resección de glándulas paratiroides identificadas en el reporte de patología de las piezas quirúrgicas. Solo uno de los pacientes tuvo lesión intencional del nervio laríngeo recurrente, debido a la presencia de un conglomerado ganglionar paratraqueal nivel VI, con invasión del nervio, envuelto en el $100 \%$ de la circunferencia; en este caso fue necesaria la resección del nervio y se hizo reconstrucción microquirúrgica del mismo. 
En los estudios imagenológicos de control, se encontró que en todos los casos se extirpó de manera exitosa la lesión, con estudio histopatológico que comprobó malignidad. Durante el período de seguimiento, en dos pacientes $(22,2 \%)$, se presentó recaída ganglionar locorregional, en niveles ganglionares diferentes, que no se habían identificado previamente, ni por estudios imagenológicos prequrúrgicos, BACAF ni hallazgos intraoperatorios. De estos, uno de los pacientes fue reintervenido utilizando la misma técnica de marcación guiada por ecografía, posterior a lo cual se obtuvo una extirpación exitosa de la lesión tumoral.

Los datos de tiroglobulina en el seguimiento estuvieron disponibles en nueve de los casos. Los niveles preoperatorios de tiroglobulina oscilaron entre 0,2 y $300 \mathrm{ng} / \mathrm{ml}$. Ocho pacientes presentaron disminución en la tiroglobulina después del procedimiento quirúrgico; en tres de los nueve casos se evidenció una adecuada respuesta bioquímica, definida como una tiroglobulina menor o igual a $2 \mathrm{ng} / \mathrm{ml}$.

Revisando los costos adicionales del procedimiento, el valor de la cirugía al utilizar esta técnica se incrementó en un promedio de $\$ 80.000$ pesos colombianos, lo cual corresponde al costo de una ecografía de tejidos blandos de cuello, mientras que, con el uso de otras técnicas, como por ejemplo ROLL, el valor adicional es de $\$ 1.600 .000$ pesos colombianos aproximadamente.

\section{Discusión}

La incidencia del carcinoma de tiroides se encuentra en aumento ${ }^{16}$. En las últimas estadísticas del GLOBOCAN $^{1}$, se registraron 567.000 casos en todo el mundo, ocupando el noveno lugar con relación a las otras neoplasias, con una tasa de incidencia global de 10,2 por cada 100.000 mujeres, representando el 5,1\% de la carga total estimada de cáncer en el género femenino. La tasa de mortalidad por esta patología es mucho menor que en otros cánceres, de 0,4 y 0,5 muertes por 100.000 personas en hombres y mujeres, respectivamente, y con alrededor de 41.000 muertes al año.

En Colombia, según los datos del Instituto Nacional de Cancerología, para el año 2018, el cáncer de tiroides en las mujeres ocupó el tercer lugar, superado por el cáncer de mama y de piel, con un total de 512 casos, representando el 11,1\% de todos los cánceres; mientras que en los hombres ocupó el séptimo lugar, con un total de 114 casos y un 3,5 \% de todos los cánceres ${ }^{17}$.

Si bien es cierto que el cáncer diferenciado de tiroides tiene en general un buen pronóstico, con una baja tasa de mortalidad, se han identificado recidivas en cuello, principalmente metástasis en los ganglios linfáticos regionales, hasta en un $30 \%{ }^{3}$. Se han descrito ciertos factores relacionados con mayor riesgo de recurrencia, como la edad menor a 15 años o mayor o igual a 55 años, género masculino, histología folicular o variedad de células altas, células en tachuela, tamaño tumoral mayor de $4 \mathrm{~cm}$, invasión tumoral local inicial y metástasis en ganglios linfáticos regionales ${ }^{18}$. En estos casos se recomienda tiroidectomía total con disección ganglionar central, con linfadenectomía uni o bilateral en quienes se documente enfermedad metastásica regional ${ }^{19}$.

En los pacientes que hayan recibido terapia de yodo 131 y que a pesar de esto se documenta persistencia de enfermedad ganglionar, está indicada la resección quirúrgica ${ }^{2}$. La reintervención del cuello debe realizarse con cautela, debido a que las dificultades durante la intervención pueden resultar en una mayor incidencia de complicaciones postoperatorias ${ }^{20,21}$. Con relación a la lesión del nervio laríngeo recurrente y de las glándulas paratiroides, las reoperaciones del compartimento central y del lecho tiroideo son más retadoras para el cirujano, si se comparan con las disecciones laterales del cuello y la tiroidectomía primaria ${ }^{10,22}$.

La otra dificultad que se presenta durante la intervención quirúrgica de un cuello previamente operado es poder identificar las lesiones metastásicas, ya que, debido al pequeño tamaño de muchas de estas, con frecuencia se hace difícil diferenciarlas del tejido cicatricial ${ }^{9,23}$. Por eso, se ha implementado la ecografía como estrategia para guiar el acto quirúrgico, con el objetivo de facilitar la localización de los ganglios linfáticos en el carcinoma recurrente de tiroides. McCoy y colaboradores describieron el uso del ultrasonido en la 
marcación preoperatoria de la lesión en pacientes con un solo ganglio linfático no palpable, al marcar la piel en el sitio donde se identificó ecograficamente el nódulo sospechoso y se encontró que el $100 \%$ de los pacientes tuvo una resección satisfactoria, mientras que en los pacientes llevados a exploración cervical sin marcación ecográfica preoperatoria la tasa de exploraciones negativas fue del $50 \%{ }^{24}$.

La planeación preoperatoria, incluido el mapeo por ultrasonido, es fundamental para identificar la ubicación de los ganglios linfáticos que van a ser resecados ${ }^{25}$; no obstante, al realizar la incisión y disección de los tejidos, con frecuencia la orientación que se tenía previa a la cirugía se pierde en el tejido cicatricial, y aunque es posible realizar la linfadenectomía, se puede pasar por alto el nódulo índice, lo cual lleva a intentos fallidos de resección ${ }^{14,24}$.

Aunque se destaca el papel de la ecografía intraoperatoria como guía para localizar las recidivas objeto de resección, aún el número de resecciones fallidas es alto ${ }^{26}$, por lo que ha surgido la necesidad de plantear una serie de estrategias con el objetivo de superar estas dificultades en el momento de abordar el cáncer de tiroides recurrente, impactando en mayor éxito quirúrgico y reduciendo las tasas de complicaciones ${ }^{12}$. Estos métodos incluyen el mapeo ecográfico preoperatorio ${ }^{25}$, la ecografía intraoperatoria ${ }^{23}$, la localización con aguja guiada por ecografía ${ }^{26}$, la inyección guiada de colorantes ${ }^{14}$ y la cirugía radioguiada ${ }^{8}$.

La cirugía radioguiada, se describió inicialmente para localizar antes de cirugía las lesiones sospechosas de malignidad no palpables en seno 27,28 . Este procedimiento depende de la acumulación de un radiotrazador en un tejido diana, lo cual facilita su identificación a través del uso de una gamma sonda portátil intraoperatoria ${ }^{29}$. Dentro de los agentes más utilizados para reintervenciones de tiroides y paratiroides se encuentran yodo131, tecnecio-99 y tecnecio-99-sestamibi ${ }^{30}$. Sin embargo, con el uso del yodo-131 es posible conseguir resultados falsos positivos, como cuando el radioisótopo es captado por el timo o las glándulas submandibulares, y falsos negativos, cuando los focos tumorales no son detectables por la gamma sonda ${ }^{31}$. La eficacia mediante la administración sistémica del radiotrazador depende de la avidez que el tumor tenga por el mismo, principalmente en carcinoma papilar no yodo ávido ${ }^{13}$.

La técnica denominada ROLL (radioguided occult lesion localization), se presenta como una alternativa óptima para superar los obstáculos durante la reintervención quirúrgica ${ }^{9}$. Este método, que se ha convertido en un estándar para varias unidades de mama en todo el mundo ${ }^{28,32}$, se ha extrapolado a individuos previamente intervenidos por carcinoma de tiroides, para facilitar la resección de lesiones recidivantes con la ayuda de una gamma sonda portátil, localizando el sitio de mayor radioactividad ${ }^{13,33}$.

Recientemente se han implementado otras estrategias, como la inyección intralesional de colorantes azules guiada por ultrasonido, pero en Colombia no se han publicado estudios con la aplicación de esta técnica para la reintervención de pacientes con recidiva de cáncer de tiroides. En nuestro estudio buscamos demostrar la utilidad de este método, con el cual se pudo identificar la lesión sospechosa mediante el ultrasonido e inyectar azul de metileno, como una estrategia que ayuda al cirujano a encontrar lesiones anormales, con el objetivo de transformar una cirugía de mayor grado de dificultad en un procedimiento más sencillo, con mejores resultados en cuanto a la resección de la recidiva y menor la tasa de complicaciones, teniendo en cuenta que se trata de una intervención en pacientes que previamente han sido operados ${ }^{7-9}$.

Encontramos varias ventajas de la técnica de inyeción intralesional de azul de metileno al compararla con la técnica de ROLL convencional. Inicialmente, se debe tener en cuenta que no todas las instituciones tienen acceso a los equipos y la tecnología que implica un servicio de medicina nuclear, y una gamma sonda portátil de alto costo es requisito para la identificación intraoperatoria de las lesiones que captan el radiotrazador. Además, para la planeación de la aplicación de la técnica de ROLL, se debe contar con la coordinación entre el especialista en cirugía y el especialista en me- 
dicina nuclear; mientras que, en nuestro estudio, el mismo cirujano fue quien realizó la marcación preoperatoria, y no se requirió de personal adicional $^{34}$.

Otro aspecto importante para resaltar es el tiempo que se requiere para la marcación previa a la cirugía. Si se utiliza el ROLL, se debe realizar la marcación con el radioisótopo al menos dos horas antes de la intervención ${ }^{3,4,13}$, mientras que en nuestro estudio se estimó un tiempo promedio de 10 minutos, lapso en el que se realizó la ecografía y la inyección del colorante azul. Este procedimiento se hizo dentro de la misma sala de cirugía y con el paciente previamente anestesiado, lo que impacta en la disminución del tiempo de marcación prequirúrgica y en toda la coordinación logística que demanda el procedimiento de medicina nuclear.

El estudio tuvo la limitación de que no se pudo realizar una comparación en cuanto a tiempos quirúrgicos, ya que no hubo ningún grupo control en el que no se utilizó la técnica, pero se estima que con el tratamiento de nuestros pacientes se redujo el tiempo operatorio al hacer el procedimiento técnicamente más fácil.

En conclusión, esta técnica quirúrgica se muestra como una estrategia segura y efectiva para la identificación intraoperatoria de las recidivas locorregionales en pacientes con carcinoma de tiroides, permitiendo una disección ganglionar exitosa orientada por la tinción, disminuyendo los riesgos de iatrogenia, el tiempo del procedimiento y los costos de la intervención, ya que soló se requiere contar con un buen equipo de ecografía y la tinción de azul de metileno, la cual es fácilmente accesible y de muy bajo costo ${ }^{14,15}$.

\section{Consideraciones éticas}

Consentimiento informado: Este estudio es una revisión de historias clínicas retrospectiva, y como tal, no hay necesidad de un consentimiento informado. El Comité de Ética Institucional aprobó el diseño y la metodología del estudio.

Declaración de conflicto de intereses: Los autores declaran no tener ningún conflicto de intereses.

Fuentes de financiación: trabajo financiado con recursos de los autores.

\section{Contribución de los autores:}

- Concepción y diseño del estudio: Ximena FrancoCastañeda, Andrés Ignacio Chala-Galindo.

- Adquisición de datos: Ximena Franco-Castañeda, Andrés Ignacio Chala-Galindo.

- Análisis e interpretación de datos: Ximena FrancoCastañeda, Andrés Ignacio Chala-Galindo.

- Redacción del manuscrito: Ximena Franco-Castañeda, Andrés Ignacio Chala-Galindo.

- Revisión crítica: Ximena Franco-Castañeda, Andrés Ignacio Chala-Galindo.

\section{Referencias}

1. Bray F, Ferlay J, Soerjomataram I, Siegel RL, Torre LA, Jemal A. Global cancer statistics 2018: GLOBOCAN estimates of incidence and mortality worldwide for 36 cancers in 185 countries. CA Cancer J Clin. 2018;68:394-424.

https://doi.org/10.3322/caac.21492

2. Haugen BR, Alexander EK, Bible KC, Doherty GM, Mandel SJ, Nikiforov YE, et al. 2015 American Thyroid Association management guidelines for adult patients with thyroid nodules and differentiated thyroid cancer: The American Thyroid Association guidelines task force on thyroid nodules and differentiated thyroid cancer. Thyroid. 2016;26:1-133.

https://doi.org/10.1089/thy.2015.0020

3. Tukenmez M, Erbil Y, Barbaros U, Dural C, Salmaslioglu A, Aksoy D, et al. Radio-guided nonpalpable metastatic lymph node localization in patients with recurrent thyroid cancer. J Surg Oncol. 2007;96:534-8. https://doi.org/10.1002/jso.20873

4. Gulcelik MA, Karaman N, Dogan L, Sahiner I, Akgul GG, Kahraman YS, Vural GU. Radioguided occult lesion localization for locally recurrent thyroid carcinoma. European Archives of Oto-Rhino-Laryngology. 2017;274:2915-9.

https://doi.org/10.1007/s00405-017-4563-2

5. Cooper DS, Doherty GM, Haugen BR, Kloos RT, Lee SL, Mandel SJ, et al. Revised American Thyroid Association management guidelines for patients with thyroid nodules and differentiated thyroid cancer. Thyroid. 2009;19:1167-214. https://doi.org/10.1089/thy.2009.0110

6. Ito Y, Tomoda C, Uruno T, Takamura Y, Miya A, Kobayashi $\mathrm{K}$, et al. Preoperative ultrasonographic examination for lymph node metastasis: usefulness when designing lymph node dissection for papillary microcarcinoma of the thyroid. World J Surg. 2004;28:498-501. https://doi.org/10.1007/s00268-004-7192-z

7. Torlontano M, Attard M, Crocetti U, Tumino S, Bruno R, Constante G, et al. Follow-up of low risk patients with papillary thyroid cancer: Role of neck ultrasonography 
in detecting lymph node metastases. J Clin Endocrinol Metab. 2004;89:3402-7.

https://doi.org/10.1210/jc.2003-031521

8. Salvatori M, Rufini V, Reale F, Gajate AM, Maussier ML, Revelli L, et al. Radio-guided surgery for lymph node recurrences of differentiated thyroid cancer. World J Surg. 2003;27:770-5. https://doi.org/10.1007/s00268-003-7014-8

9. Terzioğlu T, Senyurek YG, Tunca F, Türkmen C, Mudun A, Salmasloglu A, et al. Excision efficiency of radioguided occult lesion localization in reoperative thyroid and parathyroid surgery. Thyroid. 2010;20:1271-8. https://doi.org/10.1089/thy.2009.0441

10. Kim MK, Mandel SH, Baloch Z, Livolsi V, Langer JE, Didonato L, et al. Morbidity following central compartment reoperation for recurrent or persistent thyroid cancer. Arch Otolaryngol Head Neck Surg. 2004;130:1214-6. https://doi.org/10.1001/archotol.130.10.1214

11. Vieira-Bitencourt AG, Pereira-Lima EN, Vieira-Pinto PN, Lobato-Martins EB, Chojniak R. New applications of radioguided surgery in oncology. Clinics. 2009;64:397-402.

https://doi.org/10.1590/S1807-59322009000500005

12. Ilgan S, Oztürk E, Yildiz R, Emer O, Ayan A, Görgülü S, et al. Combination of preoperative ultrasonographic mapping and radioguided occult lesion localization in patients with locally recurrrent/persistent papillary thyroid carcinoma: A practical method for central compartment reoperations. Clin Nucl Med. 2010;35:84752. https://doi.org/10.1097/RLU.0b013e3181f48403

13. Cadena E, Bastidas F, Angarita E, Garzón J. Resección de recaídas de cáncer diferenciado de tiroides mediante cirugía radioguiada. Rev Colomb Cancerol. 2012;16:130-4. https://doi.org/10.1016/S0123-9015(12)70024-0

14. Sippel RS, Elaraj DM, Poder L, Duh QY, Kebebew E, Clark $\mathrm{OH}$. Localization of recurrent thyroid cancer using intraoperative ultrasound-guided dye injection. World J Surg. 2009;33:434-9. https://doi.org/10.1007/s00268-008-9797-0

15. Harari A, Sippel RS, Goldstein R, Aziz S, Shen W, Gosnell $J$, et al. Successful localization of recurrent thyroid cancer in reoperative neck surgery using ultrasound-guided methylene blue dye injection. J Am Coll Surg. 2012;215:555-61.

https://doi.org/10.1016/j.jamcollsurg.2012.06.006

16. Goyal RM, Jonklaas J, Burman KD. Management of recurrent cervical papillary thyroid cancer. Endocrinol Metab Clin North Am. 2014;43:565-72. https://doi.org/10.1016/j.ecl.2014.02.014

17. Instituto Nacional de Cancerología-ESE. Cáncer en cifras en el INC. Fecha de consulta: $1^{\circ}$ de agosto de 2020. Disponible en: https://www.cancer.gov.co/sites/ default/files/infografias/archivos/casos_nuevos_cancer_2018.pdf
18. Pacini F, Castagna MG. Approach to and treatment of differentiated thyroid carcinoma. Med Clin North Am. 2012;96:369-83.

https://doi.org/10.1016/j.mcna.2012.01.002

19. Sánchez G, Gutiérrez C, Valenzuela A, Tovar JR. Carcinoma diferenciado de la glándula tiroidea: hallazgos en 16 años de manejo multidisciplinario. Rev Colomb Cir. 2014;29:102-9.

20. Frasoldati A, Pesenti M, Gallo M, Caroggio A, Salvo D, Valcavi R. Diagnosis of neck recurrences in patients with differentiated thyroid carcinoma. Cancer. 2003;97:90-6. https://doi.org/10.1002/cncr.11031

21. Aksakal N, Ozturk A, Tutal F, Dogan S, Agcaoglu O, Bulakci $\mathrm{M}$, et al. Magnetic probe-guided excision of nonpalpable neck lesions: A new and safe technique for operated neck compartments. Surg Innov. 2017;24:428. https://doi.org/10.1177/1553350616676444

22. Farrag TY, Agrawal N, Sheth S, Bettegowda C, Ewertz M, Kim M, Tufano RP. Algorithm for safe and effective reoperative thyroid bed surgery for recurrent/ persistent papillary thyroid carcinoma. Head Neck. 2007;29:1069-74.

https://doi.org/10.1002/hed.20634

23. Karwowski JK, Jeffrey RB, McDougall IR, Weigel RJ. Intraoperative ultrasonography improves identification of recurrent thyroid cancer. Surgery. 2002;132:924-9. https://doi.org/10.1067/msy.2002.128478

24. McCoy KL, Yim JH, Tublin ME, Burnmeister LA, Ogilvie JB, Carty SE. Same-day ultrasound guidance in reoperation for locally recurrent papillary thyroid cancer. Surgery. 2007;142:965-72.

https://doi.org/10.1016/j.surg.2007.09.021

25. Binyousef HM, Alzahrani AS, Al-Sobhi SS, Al-Suhaibani HS, Chaudhari MA, Raef HM. Preoperative neck ultrasonographic mapping for recurrent/persistent papillary thyroid cancer. World J Surg. 2004;28:1110-4. https://doi.org/10.1007/s00268-004-7636-5

26. Zimmerman P, DaSilva M, Izquierdo R, Cico L, Kort $\mathrm{K}$, Numann P. Intraoperative needle localization during neck reexploration. Am J Surg. 2004;188:92-3. https://doi.org/10.1016/j.amjsurg.2003.11.037

27. Zurrida S, Galimberti V, Monti S, Luini A. Radioguided localization of occult breast lesions. Breast. 1998;7:113. https://doi.org/10.1016/S0960-9776(98)90044-3

28. Nadeem R, Chagla LS, Harris O, Desmond S, Thind R, Titterrell C, Audisio RA. Occult breast lesions: A comparison between radioguided occult lesion localisation (ROLL) vs. wire-guided lumpectomy (WGL). Breast. 2005;14:283-9. https://doi.org/10.1016/j.breast.2005.04.002

29. Gulec SA, Eckert M, Woltering EA. Gamma probe-guided lymph node dissection ('gamma picking') in differentiated thyroid carcinoma. Clin Nucl Med. 2002;27:859-61.

https://doi.org/10.1097/00003072-200212000-00001 
30. Ondik MP, Tulchinsky M, Goldenberg D. Radioguided reoperative thyroid and parathyroid surgery. Otolaryngol Clin North Am. 2008;41:1185-98.

https://doi.org/10.1016/j.otc.2008.07.002

31. Travagli JP, Cailleux AF, Ricard M, Baudin E, Caillou B, Parmentier C, Schlumberger M. Combination of radioiodine $\left({ }^{131} \mathrm{I}\right)$ and probe-guided surgery for persistent or recurrent thyroid carcinoma. J Clin Endocrinol Metab. 1998;83:2675-60.

https://doi.org/10.1210/jcem.83.8.5014

32. Duarte C, Bastidas F, De los Reyes A, Manrique J. Localización radioguiada de las lesiones ocultas de la mama.
Rev Colomb Cancerol. 2012;16:239-43. https://doi.org/10.1016/S0123-9015(12)70158-0

33. Giles YŞ, Sarıcı IS, Tunca F, Sormaz IC, Salmaslıoğlu A, Adalet I, et al. The rate of operative success achieved with radioguided occult lesion localization and intraoperative ultrasonography in patients with recurrent papillary thyroid cancer. Surgery. 2014;156:111626. https://doi.org/10.1016/j.surg.2014.04.012

34. Marín L, Avalos N, Valenzuela MA, Morales B. Cirugía radioguiada en hiperparatiroidismo primario: Caso clínico. Rev méd Chile. 2001;129:921-4. https://doi.org/10.4067/S0034-98872001000800012 